An Axiomatic Framework for Aggregating Weights and Weight-Ratio Matrices*

by

Jonathan Barzilai ${ }^{\dagger}$

and

Boảż Golany $y^{\ddagger}$

June 24, 1991

- Researcli supported in part by NSERC Canada grant \# A-8802.

'School of Computer Science, Technical Univ. of Nova Scotia, Halifax, N.S. B3I 2X4

:Faculty of Industrial Engineering and Management, Technion, Haifa 32000, Israel 


\begin{abstract}
In earlier work, we established an axiomatic framework for deriving consistent weight ratios from pairwise ratio matrices, using the geometric mean mapping. By adding new axioms, this framework is extended to include aggregation rules for combining weights and ratio matrices and the weighted-geometric-mean aggregation rule is shown to be consistent with this set of axioms.

In addition, by defining the output of the process as weight ratios (rather than normalized absolute weights) and using multiplicative procedures (the geometric mean and the weighted-geometric-mean aggregation rule), rank reversal is avoided.
\end{abstract}

Key Words:

Aggregation Rules, AHP, Rank Reversal, Normalization, Decision Analysis. 


\section{An Axiomatic Framework for Aggregating Weights and Weight-Ratio Matrices}

J. Barzilai and B. Golany

\section{Introduction}

We resolve Belton and Gear's rank reversal problem [4] in the Analytic Hierarchy Process (see [10]), by extending the axiomatic framework established in [1] to aggregation rules. It follows that rank reversal can be avoided if a multiplicative aggregation rule is used and normalized vectors are replaced with weight-ratio matrices.

Because it appears from Belton and Gear's discussion that the reason for the rank reversal phenomenon is improper normalization of the weight vectors, earlier work (e.g. Belton and Gear [4], Harker and Vargas [8] and Saaty and Vargas [11,12]) concentrated on

1. proposing a normalization immune to rank reversal;

2. proving that previously proposed normalizations are not immune to rank reversal;

3. legitimizing rank reversals.

More recently, the exchange $[14,6,13,9,7]$ in the March 1990 issue of Management Science is a clear indicator of the importance of resolving this controversy.

\section{The Rank Reversal Problem}

Belton and Gear consider in their Example 1 the three judgement matrices , 


$$
\left(\begin{array}{ccc}
1 & 1 / 9 & 1 \\
9 & 1 & 9 \\
1 & 1 / 9 & 1
\end{array}\right), \quad\left(\begin{array}{ccc}
1 & 9 & 9 \\
1 / 9 & 1 & 1 \\
1 / 9 & 1 & 1
\end{array}\right), \quad\left(\begin{array}{ccc}
1 & 8 / 9 & 8 \\
9 / 8 & 1 & 9 \\
1 / 8 & 1 / 9 & 1
\end{array}\right),
$$

and their corresponding normalized weight vectors

$$
\left(\begin{array}{l}
1 / 11 \\
9 / 11 \\
1 / 11
\end{array}\right), \quad\left(\begin{array}{l}
9 / 11 \\
1 / 11 \\
1 / 11
\end{array}\right), \quad\left(\begin{array}{c}
8 / 18 \\
9 / 18 \\
1 / 18
\end{array}\right)
$$

These vectors are combined (by taking their arithmetic mean) to produce the overall weight vector

$$
w^{*}=\frac{1}{3}\left(\begin{array}{c}
1 / 11 \\
9 / 11 \\
1 / 11
\end{array}\right)+\frac{1}{3}\left(\begin{array}{c}
9 / 11 \\
1 / 11 \\
1 / 11
\end{array}\right)+\frac{1}{3}\left(\begin{array}{c}
8 / 18 \\
9 / 18 \\
-1 / 18
\end{array}\right)
$$

In Example 2 they introduce an additional alternative, $D$, with judgement matrices

$$
\left(\begin{array}{cccc}
1 & 1 / 9 & 1 & 1 / 9 \\
9 & 1 & 9 & 1 \\
1 & 1 / 9 & 1 & 1 / 9 \\
9 & 1 & 9 & 1
\end{array}\right), \quad\left(\begin{array}{cccc}
1 & 9 & 9 & 9 \\
1 / 9 & 1 & 1 & 1 \\
1 / 9 & 1 & 1 & 1 \\
1 / 9 & 1 & 1 & 1
\end{array}\right), \quad\left(\begin{array}{cccc}
1 & 8 / 9 & 8 & 8 / 9 \\
9 / 8 & 1 & 9 & 1 \\
1 / 8 & 1 / 9 & 1 & 1 / 9 \\
9 / 8 & 1 & 9 & 1
\end{array}\right)
$$

and corresponding normalized weight vectors 


$$
\left(\begin{array}{l}
1 / 20 \\
9 / 20 \\
1 / 20 \\
9 / 20
\end{array}\right), \quad\left(\begin{array}{l}
9 / 12 \\
1 / 12 \\
1 / 12 \\
1 / 12
\end{array}\right), \quad\left(\begin{array}{l}
8 / 27 \\
9 / 27 \\
1 / 27 \\
9 / 27
\end{array}\right),
$$

yielding the overall weight vector

$$
w=\frac{1}{3}\left(\begin{array}{c}
1 / 20 \\
9 / 20 \\
1 / 20 \\
9 / 20
\end{array}\right)+\frac{1}{3}\left(\begin{array}{c}
9 / 12 \\
1 / 12 \\
1 / 12 \\
1 / 12
\end{array}\right)+\frac{1}{3}\left(\begin{array}{c}
8 / 27 \\
9 / 27 \\
1 / 27 \\
9 / 27
\end{array}\right) \text {. }
$$

They then observe that $w_{1}^{*}<w_{2}^{*}$ but ' $w_{1}>w_{2}$ so that the two sets of rankings are not consistent: the rank of $A$ and $B$ is reversed as a result of the inclusion of alternative $D$, even though the pairwise weight ratios associated with alternatives $A, B$, and $C$ are unchanged.

\section{No Normalization Can Prevent Rank Reversal}

By comparing equations (1) and (2), we see that the difference in relative magnitude of the components of the overall weight vectors $w^{\mathbf{x}}$ and $w$ is due to the normalization factors applied to the weight vectors. Explicitly, if the vector $y$ is obtained from the (positive) vector $x$ by the additive normalization $y_{j}=x_{j} / \sum_{i=1}^{n} x_{i}$, then any component $y_{j}$ is sensitive to changes in any other component $x_{k}$. Clearly, the same is true when a component is added to $x$. In other words, the essence of normalizing a vector is to adjust its components on 
the basis of the magnitude of other components. Since this is true for all normalizations, it follows that there does not exist any normalization which avoids rank reversal. More precisely, for any normalization, there exists a set of vectors exhibiting rank reversal. In particular, for the normalization proposed by Belton and Gear in [4] the components of the normalized vector are unchanged until a certain threshold is attained, but this normalization too, as pointed out by Saaty and Vargas in [12], is subject to rank reversal.

\section{Notation}

The matrices below are $n \times n$, vectors are $n$-dimensional, and:

1. $A=\left(a_{i j}\right)$ is a pairwise multiplicative matrix if $0<a_{i j}=1 / a_{j i}$;

2. $w=\left(w_{k}\right)$ is a multiplicative weight vector if $w_{k}>0$ and $\prod_{k=1}^{n} w_{k}=1$;

3. $C=\left(c_{i j}\right)$ is a multiplicative consistent matrix if $c_{i j}=w_{i} / w_{j}$ for some multiplicative weight vector $w$;

4. $A^{\times}, w^{\times}$and $C^{\times}$are the sets of all pairwise multiplicative matrices, multiplicative weight vectors and multiplicative consistent matrices, respectively;

5. $f^{\mathrm{x}}$ is the set of all mappings from $A^{\mathrm{x}}$ to $C^{\mathrm{x}}$;

6. $A^{\mathrm{x}}, w^{\mathrm{x}}$ and $C^{\mathrm{x}}$ are groups under componentwise multiplication and $C^{\mathrm{x}}$ is isomorphic to $w^{\mathbf{x}}$. 


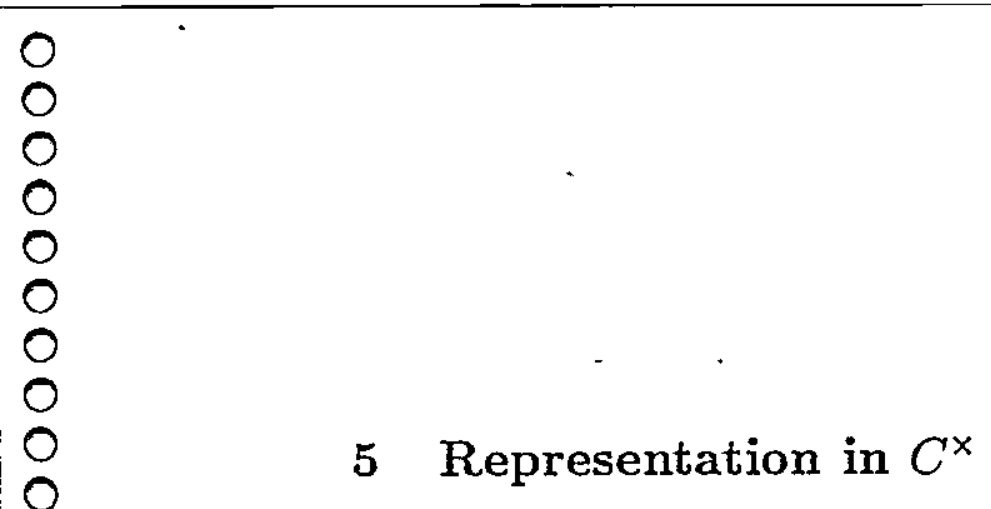

Note that the weight vectors retrieved from pairwise comparison matrices in the AHP are determined only up to a multiplicative factor. These vectors can therefore be represented by normalized proxies or, since $C^{\times}$is isomorplic to $w^{\times}$, by matrices of the form $\left(w_{i} / w_{j}\right)$. For the purpose of studying the problem of retrieving weights from pairwise comparison matrices, the choice of representation is immaterial (see e.g. [1] or [3]). However, it follows from the above that these representations are not equivalent as far as aggregating weights and ratio matrices is concerned. More importantly, for Belton and Gear's examples, weight ratios are preserved when normalized weight vectors are replaced by weight ratio matrices because no extraneous normalization factors are introduced. Indeed, the matrix representation for the examples above yields

$$
W^{*}=\frac{1}{3}\left(\begin{array}{ccc}
1 & 1 / 9 & 1 \\
9 & 1 & 9 \\
1 & 1 / 9 & 1
\end{array}\right)+\frac{1}{3}\left(\begin{array}{ccc}
1 & 9 & 9 \\
1 / 9 & 1 & 1 \\
1 / 9 & 1 & 1
\end{array}\right)+\frac{1}{3}\left(\begin{array}{ccc}
1 & 8 / 9 & 8 \\
9 / 8 & 1 & 9 \\
1 / 8 & 1 / 9 & 1
\end{array}\right)
$$

and

$$
W=\frac{1}{3}\left(\begin{array}{cccc}
1 & 1 / 9 & 1 & 1 / 9 \\
9 & 1 & 9 & 1 \\
1 & 1 / 9 & 1 & 1 / 9 \\
9 & 1 & 9 & 1
\end{array}\right)+\frac{1}{3}\left(\begin{array}{cccc}
1 & 9 & 9 & 9 \\
1 / 9 & 1 & 1 & 1 \\
1 / 9 & 1 & 1 & 1 \\
1 / 9 & 1 & 1 & 1
\end{array}\right)+\frac{1}{3}\left(\begin{array}{cccc}
1 & 8 / 9 & 8 & 8 / 9 \\
9 / 8 & 1 & 9 & 1 \\
1 / 8 & 1 / 9 & 1 & 1 / 9 \\
9 / 8 & 1 & 9 & 1
\end{array}\right)
$$

The relative ranking of alternatives $A$ and $B$ is unchanged since the numeriçal value of $w_{1} / w_{2}$ is preserved - in fact, $W^{*}$ is a principal minor of $W$ (that is, $W^{*}$ is obtained by deleting certain rows and the same numbered columns of $W$ ). 


\section{Axioms for Aggregation Rules}

Let $F$ be an aggregation rule for combining $l$ positive weights and $n \times n$ matrices. This rule is then a mapping from the set of all $\left\{\lambda_{1}, \ldots, \lambda_{l} ; A_{1}, \ldots, A_{l}\right\}$ to the set of positive $n \times n$ matrices.

Guided by the examples above and the underlying multiplicative structures, we postulate axioms for aggregation rules and prove that the weighted-geometric-mean aggregation rule satisfies these axioms and, consequently, is immune to rank reversal.

Axiom 1. The aggregation rule $F$ satisfies

$$
F\left(\lambda_{1}, \ldots, \lambda_{l} ; P\left(A_{1}\right), \ldots, P\left(A_{l}\right)\right)=P\left(F\left(\lambda_{1}, \ldots, \lambda_{l} ; A_{1}, \ldots, A_{l}\right)\right),
$$

where the operator $P$ denotes taking a (fixed) principal minor of the appropriate matrices.

Axiom 2. If the input matrices of $F$ are consistent, so is its output matrix -

$$
A_{k} \in C^{\times} k=1, \ldots, l \Rightarrow F\left(\lambda_{1}, \ldots, \lambda_{l} ; A_{1}, \ldots, A_{l}\right) \in C^{\times}
$$

Axiom 3. For some $f \in f^{x}$,

$$
F\left(\lambda_{1}, \ldots, \lambda_{l} ; f\left(A_{1}\right), \ldots, f\left(A_{l}\right)\right)=f\left(F\left(\lambda_{1}, \ldots, \lambda_{l} ; A_{1}, \ldots, A_{l}\right)\right)
$$

The significance of Axiom 1 is that if $P(A)$ is a principal minor of $A$, then $P(A)$ and $A$ represent the same judgment ratios over the objects they have in common. It follows directly from the definition that if $F$ satisfies Axiom 1, the weight ratios of the common objects 
are identical and therefore not affected by the inclusion of additional objects. Formally:

Theorem. An aggregation rule satisfying Axiom 1 is not subject to rank reversal.

Axiom 2 is needed since an arbitrary aggregation rule may produce matrices which are neither consistent nor even pairwise multiplicative, as is the case for $W^{*}$ and $W$ in $\left(1^{\prime}\right)$ and $\left(2^{\prime}\right)$ above. That the arithmetic mean destroys this property is not a surprise in view of our earlier analysis of the underlying algebraic structure (see Barzilai et al. $[1,2,3]$ ).

Axiom 3 means that if the input pairwise multiplicative matrices are converted - using the mapping $f$ - to their consistent representative matrices and the resulting multiplicative consistent matrices are aggregated using the rule $F$, the result obtained is the same as when the input pairwise multiplicative matrices are first aggregated using the rule $F$ and the pairwise multiplicative matrix obtained in this manner is then converted to its consistent representative matrix using the mapping $f$. In other words, the final result is independent of the order of operation.

Keeping in mind the underlying multiplicative structures, it is easy to extend the observations in $\S 5$ :

Theorem. The weighted-geometric-mean aggregation rule ${ }^{\dagger}$

$$
F\left(\lambda_{1}, \ldots, \lambda_{l} ; A_{1}, \ldots, A_{l}\right)=\prod_{k=1}^{l} A_{k}^{\lambda_{k}}
$$

satisfies Axioms 1-3.

Proof. For objects $i$ and $j$ belonging to the principal minor corresponding to the operator

${ }^{\dagger}$ All operations are carried out componentwise. 
$P$, both sides of the equation defining Axiom 1 are given by

$$
\prod_{k=1}^{l}\left(a_{i j}\right)_{k}^{\lambda_{k}}
$$

where $\left(a_{i j}\right)_{k}$ denotes the $i j$ element of $A_{k}$. Therefore, $F$ satisfies Axiom 1.

- Next, note that for $m=1, \ldots, l, A_{m} \in C^{\mathrm{X}}$ is equivalent to $\left(a_{i j}\right)_{m}\left(a_{j k}\right)_{m}\left(a_{k i}\right)_{m}=1$. This implies

$$
\prod_{m=1}^{l}\left(a_{i j}\right)_{m}^{\lambda_{m}} \prod_{m=1}^{l}\left(a_{j k}\right)_{m}^{\lambda_{m}} \prod_{m=1}^{l}\left(a_{k i}\right)_{m}^{\lambda_{m}}=1,
$$

so that $F\left(\lambda_{1}, \ldots, \lambda_{l} ; A_{1}, \ldots, A_{l}\right)$ is consistent. Hence $F$ satisfies Axiom 2.

Finally, in conjunction with the geometric mean mapping defined by

$$
f(A)=W=\left(w_{i j}\right) \quad w_{i j}=\left(\frac{\prod_{k=1}^{n} a_{i k}}{\prod_{k=1}^{n} a_{j k}}\right)^{1 / n},
$$

the weighted-geometric-mean aggregation rule satisfies Axiom 3 since

$$
\prod_{m=1}^{l}\left(\frac{\prod_{k=1}^{n}\left(a_{i k}\right)_{m}^{1 / n}}{\prod_{k=1}^{n}\left(a_{j k}\right)_{m}^{1 / n}}\right)^{\lambda_{m}}=\prod_{k=1}^{n}\left(\frac{\prod_{m=1}^{l}\left(a_{i k}\right)_{m}^{\lambda_{m}}}{\prod_{m=1}^{l}\left(a_{j k}\right)_{m}^{\lambda_{m}}}\right)^{1 / n} .
$$

To illustrate the above, the weighted-geometric-mean produces for the Belton and Gear examples the matrices

$$
U^{*}=\left(\begin{array}{ccc}
1 & 1 / 9 & 1 \\
9 & 1 & 9 \\
1 & 1 / 9 & 1
\end{array}\right)^{1 / 3} \times\left(\begin{array}{ccc}
1 & 9 & 9 \\
1 / 9 & 1 & 1 \\
1 / 9 & 1 & 1
\end{array}\right)^{1 / 3} \times\left(\begin{array}{ccc}
1 & 8 / 9 & 8 \\
9 / 8 & 1 & 9 \\
1 / 8 & 1 / 9 & 1
\end{array}\right)^{1 / 3}
$$

and

$$
U=\left(\begin{array}{cccc}
1 & 1 / 9 & 1 & 1 / 9 \\
9 & 1 & 9 & 1 \\
1 & 1 / 9 & 1 & 1 / 9 \\
9 & 1 & 9 & 1
\end{array}\right)^{1 / 3} \times\left(\begin{array}{cccc}
1 & 9 & 9 & 9 \\
1 / 9 & 1 & 1 & 1 \\
1 / 9 & 1 & 1 & 1 \\
1 / 9 & 1 & 1 & 1
\end{array}\right)^{1 / 3} \times\left(\begin{array}{cccc}
1 & 8 / 9 & 8 & 8 / 9 \\
9 / 8 & 1 & 9 & 1 \\
1 / 8 & 1 / 9 & 1 & 1 / 9 \\
9 / 8 & 1 & 9 & 1
\end{array}\right)^{1 / 3}
$$


$U^{*}$ and $U$ are (consistent) pairwise multiplicative and $U^{*}$ is a principal minor of $U$. Thus, the ranking of alternatives $A, B$ and $C$ under $U^{*}$ and $U$ is identical.

\section{Conclusions}

By adding new axioms, the axiomatic framework for deriving consistent weight ratios from pairwise ratio matrices developed in [1] has been extended to deal with aggregation rules for combining weights and ratio matrices.i While more work needs to be done to further extend these results to a general hierarchy, this axiomatic framework has already enabled us to resolve the Belton and Gear rank reversal problem.

\section{References}

[1] J. Barzilai, W.D. Cook and B. Golany, "Consistent Weights for Judgements Matrices of the Relative Importance of Alternatives," Openations Research Letters, 6(3), 131-134; 1987 .

[2] — "The Analytic Hierarchy Process: Structure of the Problem and Its Solutions," in Extremal Methods and Systems Analysis II, A. Ben-Israel, A. Ben-Tal, B. Golany, K.O. Kortanek and J.J. Rosseau, Eds., Greenwood Press, to appear.

[3] J. Barzilai and B. Golany, "Deriving Weights from Pairwise Comparison Matrices: The Additive Case," Operations Research Letters, 9(6), 407-410, 1990.

[4] V. Belton and T. Gear, "On a Shortcoming of Saaty's Method of Analytic Hịerarchies," Omega, 11(3), 228-230, 1983. 
[5] _ , "The Legitimacy of Rank Reversal - a Comment," Omega, 13(3), 143-144, 1985.

[6] J.S. Dyer, "Remarks on the Analytic Hierarchy Process," Management Science, 36(3), 249-258, 1990.

[7] J.S. Dyer, "A Clarification of 'Remarks on the Analytic Hierarchy Process'," Management Science, 36(3), 274-275, 1990.

[8] P.T. Harker and L.G. Vargas "The Theory of Ratio Scale Estimation: Saaty's Analytic Hierarchy Process," Management Science, 33(11), 1383-1403, 1987.

[9] P.T. Harker and L.G. Vargas, "Reply to 'Remarks on the Analy tic Hierarchy Process' by J.S. Dyer," Management Science, 36(3), 269-273, 1990.

[10] T.L. Saaty, The Analytic Hiemarchy Process, McGraw-Hill, 1980.

[11] T.L. Saaty and L.G. Vargas "Inconsistency and Rank Preservation," Journal of Mathematical Psychology, 28(2), 205-214, 1984.

[12] , "The Legitimacy of Rank Reversal," Omega, 12(5), 513-516, 1984.

[13] T.L. Saaty, "An Exposition of the AHP in Reply to the Paper 'Remarks on the Analytic Hierarchy Process'," Management Science, 36(3), 259-268, 1990.

[14] R.L. Winkler, "Decision Modeling and Rational Choice: AHP and Utility Theory," Management Science, 36(3), 247-248, 1990. 